Revue de l'Institut des langues et cultures

d'Europe, Amérique, Afrique, Asie et Australie

$20 \mid 2014$

Le conte : d'un art à l'autre

\title{
Les contes des frères Grimm chez Goldschmidt : l'imaginaire d'une enfance menacée
}

The Grimm Brothers' Fairy Tales in the Works of Goldschmidt: Fantasies of a Threatened Childhood

Die Märchen der Gebrüder Grimm bei Goldschmidt: Die Vorstellungswelt eines verfolgten Kindes

Katja Meister

\section{(2) OpenEdition}

Journals

Édition électronique

URL : http://journals.openedition.org/ilcea/2692

DOI : 10.4000/ilcea.2692

ISSN : 2101-0609

Éditeur

UGA Éditions/Université Grenoble Alpes

Édition imprimée

ISBN : 978-2-84310-290-5

ISSN : 1639-6073

Référence électronique

Katja Meister, « Les contes des frères Grimm chez Goldschmidt : l'imaginaire d'une enfance

menacée », ILCEA [En ligne], 20 | 2014, mis en ligne le 08 décembre 2014, consulté le 10 décembre

2020. URL : http://journals.openedition.org/ilcea/2692 ; DOI : https://doi.org/10.4000/ilcea.2692

Ce document a été généré automatiquement le 10 décembre 2020.

(c) ILCEA 


\title{
Les contes des frères Grimm chez Goldschmidt : l'imaginaire d'une enfance menacée
}

\author{
The Grimm Brothers' Fairy Tales in the Works of Goldschmidt: Fantasies of a \\ Threatened Childhood \\ Die Märchen der Gebrüder Grimm bei Goldschmidt: Die Vorstellungswelt eines \\ verfolgten Kindes
}

Katja Meister

Au départ, c'est l'Allemagne du XIX ${ }^{e}$ siècle, celle des sapins de Noël et des contes de Grimm, [...] mais déjà prise dans la gangue du crime et de l'horreur nazis. (La Traversée des fleuves, quatrième de couverture)

C'est ainsi que les éditions du Seuil présentent les origines de leur auteur sur la quatrième de couverture de son autobiographie $L a$ Traversée des fleuves. Certes, cette citation se situe à la croisée de la critique littéraire et des arguments de vente, mais nous sommes d'emblée frappés par l'entrée en matière évoquant les «contes de Grimm». Les contes des frères Grimm, tout comme les «sapins de Noël» sont-ils à considérer comme des mots-clés censés intéresser un public plus large, employés dans un but commercial? On les présente comme un des éléments caractéristiques de l'Allemagne du XIX ${ }^{e}$ siècle, alors que le pays serait «déjà » sous l'emprise du «crime» et de «l'horreur nazis ». Une lecture plus précise établira que Goldschmidt postule une relation assez complexe et parfois contradictoire entre la culture germanique et le nazisme : face aux crimes contre l'humanité, le xIx ${ }^{e}$ siècle n'est pas «innocent»; la culture germanique serait à reconsidérer sous un nouvel angle, celui des violences perpétrées durant le siècle suivant. Goldschmidt a dit que l'Allemagne a été le premier pays " occupé » par les nazis ${ }^{1}$, mais on trouve également des citations qui laissent croire qu'une certaine tradition issue du XIX ${ }^{e}$ siècle, représentée par des épigones du romantisme, aurait mené à la catastrophe du nazisme et qu'il n'existe point de tradition culturelle qui ne soit mêlée aux crimes du $\mathrm{xx}^{\mathrm{e}}$ siècle d'une manière ou d'une autre². 
2 Dans cette contribution, nous tenterons de démontrer que les Contes des frères Grimm constituent une référence à la fois biographique et littéraire au cœur de l'écriture de Georges-Arthur Goldschmidt, pourtant rarement étudiée sous cet angle ; une référence d'autant plus ambivalente que les contes lui permettent à la fois d'évoquer un imaginaire enfantin à travers des scènes de lecture et de le transgresser dans une démarche purement littéraire, qu'elle soit autobiographique ou fictive. Dans la longue série de livres d'inspiration autobiographique, seul le premier ouvrage est désigné comme relevant du genre romanesque (Le Miroir quotidien), tous les autres sont désignés comme des récits (Un jardin en Allemagne, La Forêt interrompue...) et par le terme d'«Erzählung » s'ils sont publiés en allemand (Die Absonderung, Die Aussetzung...), pour rendre compte de la part imaginaire. À plusieurs reprises, l'auteur revendique le terme d'autofiction pour décrire sa démarche littéraire.

\section{I. Évocation des faits historiques au crible de la référence aux Contes de Grimm}

3 Goldschmidt insiste sur l'omniprésence de l'univers des contes des frères Grimm dans sa perception enfantine qu'il retrouve «à sa porte ", dans la nature qui entoure la maison familiale; pourtant, cette évocation de la forêt, lieu de toutes sortes d'aventures et du merveilleux, n'aboutit pas à un ré-enchantement permanent au contact du paysage, mais à la crainte d'être « mis à mort » qui ne saurait être dissociée de ce semblant d'idylle :

Je retrouvais les paysages de contes de Grimm à ma porte, la forêt sombre, les étangs mystérieux, entourés d'arbres aux épais feuillages, et en hiver le dessin étrange des branches, sans cesse agitées par le vent. Un effroi indéfinissable habitait désormais cette campagne si calme, doucement étendue. Partout on pouvait y être mis à mort. (La Traversée des fleuves, p. 111)

4 La menace de la mort est un des éléments constitutifs de l'enfance de Georges-Arthur Goldschmidt, qu'il ne cesse de raconter dans ses récits d'enfance. Tout comme la Bible, les contes des frères Grimm, dont Ludwig Richter a donné une célèbre version, constituent une des lectures fondatrices de l'imaginaire enfantin selon Goldschmidt ${ }^{3}$. Dans la Bible, le jeune garçon Jürgen Arthur découvre à la fois Jésus et le mot « juif » ainsi que la figure du martyre qui le préoccupera des années durant, tandis que les contes des frères Grimm le plongent au cœur d'une culture germanique dont il sera exclu par les Lois de Nuremberg.

5 La lecture des contes établit un lien avec le passé littéraire du XIXe siècle et permet de mettre en scène l'arbitraire de la menace de mort dont il est la cible. Dans un autre texte autobiographique, Une vie entre la France et l'Allemagne, l'auteur cite les Contes des frères Grimm comme un des éléments de la " germanité » caractéristique de l'identité familiale, de leur sentiment d'appartenance au peuple allemand dont ils seraient l'incarnation exemplaire, vivant pour ainsi dire aux côtés de Ludwig Richter, de Hans Thoma et des frères Grimm ${ }^{4}$.

6 Toujours dans La Traversée des fleuves, on retrouve cette citation qui cerne bien son profond sentiment d'appartenance à la culture germanique et une répulsion liée à la menace de mort émanant de la nation allemande. Évoquer les contes permet donc à l'auteur d'illustrer l'écart entre l'image qu'il a de lui-même et les catégories raciales établies par les lois de Nuremberg, sans citer ces dernières.

Les mélodies, les images, les contes, ma situation même au sein de cet environnement allemand du $\mathrm{XIX}^{\mathrm{e}}$ siècle m'avaient imprégné jusqu'au plus intime de 
mon être. Il n'était peut-être guère possible d'être un enfant plus allemand que je ne l'étais alors. En même temps je sentais peser, issue de cela même qui m'avait ainsi marqué, une formidable et barbare menace, étrangère et familière tout à la fois. Une présence opaque ne cessait de geler toute chose. (La Traversée des fleuves, p. 111)

7 La toute première fonction des contes des frères Grimm dans les récits de Goldschmidt est donc de restituer une situation historique précise et d'illustrer la contradiction marquant le jeune garçon qui se trouve progressivement exclu de la société au point de craindre pour sa survie, alors que certaines références culturelles de cette même société ont formé son esprit. Vu son âge à l'époque - Goldschmidt avait onze ans lorsqu'il a quitté ses parents en 1939 pour trouver un premier refuge en Italie avant d'arriver en France l'exemple des contes paraît particulièrement adapté à son sujet, puisqu'il fait partie d'un univers enfantin, des références littéraires étroitement liées à des scènes de sa vie familiale ${ }^{5}$.

8 Ses écrits d'inspiration autobiographique, saturés de références littéraires plus ou moins voilées (Rousseau et Karl Philipp Moritz, Peter Weiss et Peter Handke, pour n'en citer que quelques-unes), rendent compte de l'imaginaire d'un enfant menacé de persécution raciale et d'extermination. Les contes servent à restituer la manière de penser de l'enfant qu'il était ou qu'il aurait pu être, alors que l'auteur connaît évidemment et commente d'ailleurs les hypothèses émises en sciences sociales sur le nazisme et la persécution des Juifs ${ }^{6}$. Dans cette perspective, on peut ajouter que l'évocation des contes rétablit le point de vue de l'enfant dans un texte littéraire, par rapport à une pensée nourrie de réflexions théoriques de l'auteur devenu adulte. Le jeune garçon de ces récits ignore pourquoi il doit quitter sa famille et s'imagine une raison bien plus concrète et charnelle à l'origine de son départ: la découverte de la sexualité et une certaine fascination pour les punitions dès son plus jeune âge ${ }^{7}$. L'auteur dira à maintes reprises à propos de cet imaginaire narcissico-masochiste qu'il l'a sauvé du pire, en lui permettant de tenir à distance la douleur de la séparation et la nostalgie de la terre natale, en le confinant dans un univers clos et, d'une certaine manière, maîtrisable, celui d'un internat en Haute-Savoie où des châtiments corporels sont imposés. Au lieu de se sentir anéanti par ces derniers, l'enfant en détournera la signification et en tirera la preuve de son existence.

9 Les rêveries masochistes font des récits de Georges-Arthur Goldschmidt un cas à part dans les témoignages littéraires de la Shoah, par l'omniprésence de ses propres fantasmes qui l'emportent sur le thème de la persécution. Comme personne avant lui, Goldschmidt nous montre une victime de la persécution raciale qui accueille les agressions des autres. L'auteur Goldschmidt met en scène un enfant qui se livre corps et âme à des sentiments susceptibles de heurter ses lecteurs au lieu de les bercer dans une empathie confortable et un sentiment de compassion envers l'enfant persécuté qui leur permettraient de se décharger de leur propre responsabilité historique. Dans les récits de Goldschmidt, la victime est coupable de ses propres pensées et de ses propres actes déviants (supposés, imaginés ou réels); il s'agit d'une perspective déroutante mais en parfait accord avec certains résultats de la recherche psychiatrique et psychologique ${ }^{8}$. Ceci dit, un enfant qui se sent coupable de ses propres pensées sexuelles peut toujours susciter de la compassion, et par ailleurs, le masochisme n'est qu'une des issues qui s'offrent aux hommes, mais il nous semble important de souligner que les agressions que ressent le personnage-enfant du récit de Goldschmidt ne sont pas à considérer comme émanant de sa personnalité, mais comme une réaction à une situation menaçante et oppressante qui finit par former la personnalité de l'enfant. Une citation intéressante précise que la culpabilité et 
l'innocence vont toujours de pair, et présente ce mélange de culpabilité et d'innocence, caractéristique du personnage principal de Goldschmidt, par rapport à des protagonistes issus des Contes de Grimm :

Il y avait une fois un roi, «Es war einmal ein König »... Ce roi, c'était lui. On était toujours à la fois le roi et le jeune homme beau et pauvre, on en était l'innocence et on était aussi le roi coupable. (Le Miroir quotidien, p. 141)

Toujours dans un souci de restituer le cadre historique, Goldschmidt rappelle que le volume des Contes de Grimm est l'un des rares objets que le jeune garçon, personnage de ses récits, emporte avec lui au moment de la séparation d'avec ses parents qui s'avère définitive. La présence matérielle du recueil de contes, de cet objet issu de la maison natale, crée un lien entre son foyer et l'exil :

Il avait encore pu emporter quelques beaux livres illustrés : Contes, Nouvelles, Dans la forêt et sur la lande de Hermann Löns, la vie de Johann-Hinrich Wichern [...].

Dans son casier il était aussi autorisé à ranger des cahiers, des porte-plume et une bouteille d'encre Waterman ainsi qu'une petite voiture Märklin de plus en plus usée. Il passait parfois sa main dessus, peut-être suffisait-il alors de fermer les yeux pour être encore à la maison. [Ma traduction.] (Absonderung, p. 66-67) ${ }^{9}$

11 Ici, les Märchen ne sont certes pas explicitement attribués aux frères Grimm $^{10}$, mais une autre occurrence dans Le Miroir quotidien fait référence à une ancienne édition des Contes de Grimm que le jeune homme possédait jadis :

Cette fois, il s'assit sur le lit pour oublier ces objets trop familiers et il ouvrit sa valise pour en sortir le petit volume des Contes de Grimm. Ce n'était plus

l'exemplaire ancien, mais un livre à la couverture grise. (Le Miroir quotidien, p. 140)

L'édition évoquée dans ce récit est achetée après la guerre dans une gare allemande, détail significatif si l'on se rappelle que la gare principale d'Hambourg est le lieu de la séparation d'avec ses parents que Goldschmidt ne cesse de raconter :

Il prit sur son étagère l'exemplaire des Contes de Grimm. Il l'avait acheté un jour dans une gare allemande, très haute, pleine de vent et de monde. (Le Miroir quotidien ,p. 81)

\section{Les Contes de Grimm comme (pré)texte : une clé dans l'expérience de la persécution}

13 Dans Le Miroir quotidien, le premier récit à résonance autobiographique de Goldschmidt, les contes font écho à la situation du jeune homme, personnage principal du récit et alter ego de l'auteur, et permettent de saisir sa prédisposition psychologique à travers les contes dont la lecture le touche particulièrement, et cela ne semble pas relever du hasard si les contes cités par Goldschmidt sont parmi les plus cruels du recueil des Kinder- und Hausmärchen. Il s'agit notamment de "L'enfant entêté" [ "Das eigensinnige Kind» (KHM 117)] et des deux contes suivants uniquement présents dans la toute première édition de 1812: «La Main au couteau» [ «Die Hand mit dem Messer» (KHM 8a)] et "Comment des enfants ont joué ensemble au boucher» ["Wie die Kinder Schlachtens miteinander gespielt haben $\gg(\text { KHM 22a) }]^{11}$.

14 Le procédé narratif consiste à insérer dans le récit un conte intégralement traduit, dont certains points essentiels reprennent des sujets qui préoccupent l'alter ego de Goldschmidt - les notions de crime et de châtiment apparaissent dans tous les contes cités ; et de prétendre que la lecture du conte à l'intérieur du récit-cadre serait due à un hasard. Il s'agit bien évidemment d'un choix de l'auteur. Par ailleurs, il aurait fallu que le jeune 
personnage ait sous la main une édition regroupant la toute première édition de 1812 et les modifications de 1815 afin de découvrir tous les contes cités, ce qui n'est certes pas exclu, mais peu probable. Qui plus est, le personnage dit avoir découvert «La Main au couteau » par l'intermédiaire d'un surveillant de l'internat qui lui aurait lu le conte. Or, à notre connaissance, le conte n'avait pas été traduit en français à cette époque, et de toute façon, la traduction donnée est sans aucun doute de la main de l'auteur, connu pour ses traductions littéraires avant de publier les récits qui ont fondé sa renommée ${ }^{12}$.

15 À l'origine de la culpabilité évoquée par le personnage principal du Miroir quotidien se trouve le sentiment de culpabilité ressenti par un survivant de l'Holocauste alors que tant d'autres ont péri ; le fait de se rendre coupable par ses actes contribue de toute évidence à renforcer et à aiguiser son sentiment de culpabilité :

Il n'avait cessé de se sentir fautif : tant d'autres étaient morts qui n'avaient que ses origines et lui survivait et ne cessait de se rendre coupable: les moments de culpabilité prenaient le corps de bas en haut. (Le Miroir quotidien, p. 55)

Il faut souligner la distance entre l'auteur devenu adulte et l'enfant mis en scène dans les récits, avec la part de reconstruction littéraire et critique que cela suppose. Le «il » du Miroir quotidien est anonyme, et ce n'est que deux décennies plus tard, avec la parution de l'autobiographie La Traversée des fleuves en 1999, que l'on peut tenter de discerner ce qui, dans ce roman, relève de l'autobiographie et ce qui relève de la fiction, si l'on adhère à l'hypothèse que l'autobiographie La Traversée des fleuves présente un vrai texte de référence, moins fantasmé et plus véridique que les récits de l'auteur.

Les contes opèrent une mise à distance par le récit : dans les contes insérés, les sujets (le motif d'une séparation qui s'avère définitive, le motif d'une fausse accusation, les notions de crime et de châtiment, pour n'en citer que quelques-uns) du récit-cadre reviennent sur un autre mode, et sont évoqués par rapport à d'autres personnages. "La Main au couteau » met en scène une fausse accusation, portée par l'elfe contre la fillette et qui est à l'origine de leur séparation définitive. Dans le conte, le bon elfe est amoureux de la fillette et l'aide à accomplir les lourdes tâches imposées par sa mère jusqu'à ce que les frères de la fillette attirent l'elfe, l'appellent comme elle avait coutume de le faire et lui coupent une main. Cette constellation évoque implicitement la situation historique : l'exil qui s'avère définitif et toutes les confusions qui règnent autour de cet exil, et l'on pourra même penser au fait que les Juifs ont été faussement accusés par les nazis de nuire à la nation allemande. Ces pistes d'interprétation ne sont pas données dans le texte; au contraire, le personnage prétend ne pas comprendre pourquoi le conte le met mal à l'aise : "Pourquoi donc avait-il tant rougi lorsqu'un surveillant lui avait lu ce conte de Grimm intitulé "La Main au couteau" ?» (Le Miroir quotidien, p. 56).

L'auteur incite donc ses lecteurs à construire eux-mêmes le nouveau sens que prennent les Contes après l'extermination des Juifs européens. Cette relecture est déclenchée par les réflexions de son personnage principal à la suite de la lecture du conte «La Main au couteau ", réflexions en décalage avec le récit. En effet le personnage évoque son propre sentiment d'innocence, libérateur ${ }^{13}$, tandis que la fillette du conte est certes innocente, mais vouée à une vie solitaire et laborieuse à cause de la fausse accusation dont elle est la cible. Ce sont les frères malintentionnés de la fillette qui ont coupé la main de l'elfe mais celui-ci croit sa bien-aimée coupable et la quitte à jamais. Par conséquent, pour la fillette du conte, savoir qu'elle n'y est pour rien dans la disparition de l'elfe n'ira pas de pair avec un sentiment de soulagement, et par ailleurs, le conte n'évoque nulle part son sentiment d'innocence. 
19 À première vue, le procédé de réécriture semble se limiter à traduire le texte, mais en réalité, l'auteur incite ses lecteurs à adopter une nouvelle perspective et à chercher les liens entre le conte inséré dans le récit et les faits historiques évoqués, à travers le questionnement de son personnage principal.

20 Une légende espagnole insérée dans le récit du Miroir quotidien évoque également le thème d'une séparation imposée et d'un retour impossible sur les lieux de l'enfance, toujours mêlés au motif d'une incompréhension entre les protagonistes qui s'avère fatale. D'après cette légende, « Le jeune berger et le serpent » dont l'origine n'est pas révélée par l'auteur, un jeune berger qui a nourri un serpent durant des années, est tué par ce même serpent lorsque, de retour de son service militaire, il reprend le chemin de jadis :

Il siffla comme jadis, pour [...] appeler [le serpent] et l'animal sortit de son trou. Il était devenu énorme et sifflait de colère comme pour dire «Pourquoi m'as-tu abandonné ?». Puis il se jeta sur le jeune homme, l'entoura de ses anneaux et le broya. (Le Miroir quotidien, p. 111)

21 Pour le personnage principal du récit, cette légende est liée à un sentiment de honte profondément ancré en lui, et il l'associe à un évènement honteux, réellement vécu par lui : un jour pendant la guerre, il fit une chute par peur d'être surpris nu en pleine nature. Or, il portait six bouteilles de lait, si précieuses à cette époque, qu'il était censé rapporter à l'internat. De retour là-bas, il inventa une histoire de chute en montagne pour se justifier. Peu après, un surveillant lui aurait raconté ce récit enchâssé, et la légende Le jeune berger et le serpent serait resté imprégnée de la honte ressentie auparavant. Pourtant, rien dans la légende ne permet d'établir de lien avec un sentiment de honte, si ce n'est à travers les thèmes de l'exil, du retour impossible et de la fausse accusation. Là encore, c'est au lecteur qu'incombe la tâche d'interpréter l'insertion du conte dans le récit pour combler un blanc du récit-cadre.

22 Ce sont les réflexions du personnage principal qui amorcent le nouveau sens que prennent les contes. Après le passage où est insérée cette légende, le récit parle d'un sentiment oppressant de honte ( La honte de cette enfance qui lui revenait sans cesse lui fermait la poitrine »), avant de rappeler la menace de l'arrestation : «L'horreur d'être luimême [et non pas quelqu'un d'autre, non menacé du fait de ses origines] était si forte qu'il se prit la tête à deux mains : comment se faisait-il qu'on ne l'eût pas encore arrêté ?» (Le Miroir quotidien, p. 111-112)

Un effet complémentaire à la mise en distance produite par le récit enchâssé est l'omniprésence des sujets qui préoccupent le personnage principal du Miroir quotidien : il ne peut échapper à ses pensées; tout ce qu'il touche est imprégné par ses fantasmes. Cet effet nous paraît prédominant dans le choix d'évoquer "L'Enfant entêté ».

Le jeune homme du roman cherche à se distraire de ses propres actes (c'est-à-dire de l'auto-érotisme) et de ses pensées jugées coupables, et, dans les contes de Grimm, il tombe sur « un conte très court, qu'il n'avait jamais lu [...]». Peut-être est-ce le titre qui l'avait frappé: «L'Enfant entêté » (Le Miroir quotidien, p. 81). C'est l'histoire d'un enfant qui s'oppose à sa mère et meurt ensuite d'une maladie incurable, car son comportement déplait à Dieu. Pourtant, l'enfant persiste dans son obstination, et son petit bras sort de sa tombe jusqu'à ce que sa mère le batte à coups de verges et que l'enfant trouve enfin le repos. L'effet produit par ce conte sur le personnage-lecteur est tout le contraire de la distraction tant recherchée. Renvoyé à ses propres fantasmes, il reste figé et tente de dissimuler son corps, ainsi que sa présence, et son identité. Sans qu'on puisse déterminer 
aux yeux de qui il souhaite disparaître, il fait comme s'il n'existait pas et semble ne pas vouloir sentir « sa propre présence » :

Comme s'il avait été frappé de paralysie, il se mit debout, le corps figé, les jambes raides. Il était décidé à ne plus regarder que droit devant lui. Il marcha en tentant de poser les pieds le moins possible par terre, pour ne pas remarquer sa propre présence. Il ne fallait pas qu'on le surprît ainsi à être LUI-MÊME. (Le Miroir quotidien, p. 82)

On retrouve ici certes les notions de crime et de châtiment, communes à tous les contes insérés, mais symboliquement très rapprochées des pratiques considérées comme coupables : d'abord à travers le bras de l'enfant, symbole de son obstination. Les mains, prolongement du bras, sont l'élément du corps sur lequel se dirige tout particulièrement l'attention des éducateurs: nous venons de citer le soulagement que le jeune homme ressent lorsqu'il peut montrer ses mains en signe d'innocence (soulagement mentionné juste après la lecture de « La Main au couteau »). En outre, la punition corporelle, infligée par une figure féminine et maternelle, semble nécessaire pour permettre le repos de l'enfant et fait écho aux punitions infligées par la directrice de l'internat en Haute-Savoie et l'apaisement que le jeune interne ressent dans cette souffrance physique ${ }^{14}$. Par ailleurs, dans les deux autres contes des frères Grimm cités par l'auteur, le crime est également associé aux mains : dans "La Main au couteau », le titre à lui seul renvoie aux frères devenus criminels par leur acte, et c'est également la main de l'elfe qui est coupée. Les mains, instrument du meurtre commis en toute innocence enfantine, sont implicitement présentes dans la légende "Comment des enfants ont joué ensemble au boucher »: le couteau avec lequel l'enfant, à qui a été dévolu le rôle du boucher, tue l'enfant désigné comme cochon, doit être tenu par sa ou ses mains, tout comme le plat dans lequel la petite fille « aide cuisinière » recueille le sang.

Le Miroir quotidien accorde une place centrale à l'un des contes des frères Grimm : grâce à la lecture du conte «Comment des enfants ont joué ensemble au boucher», traduit par Goldschmidt et inséré intégralement dans son récit, le personnage principal est libéré de son sentiment de culpabilité. Au conte succède une vague de questionnements relative à «la honte»: la lecture du conte déclenche une forte réaction psychique chez le personnage qui fait l'objet d'un récit propre sans qu'on puisse parler d'«adaptation » stricto sensu.

Un effet paradoxal, déjà amorcé dans la légende - le choix de la pomme est le symbole de l'innocence des enfants qui ne connaissent pas encore la valeur de l'argent - est la perversion du symbole de la pomme, puisque la pomme est présentée dans Le Miroir quotidien comme un des éléments d'une mise en scène sexuellement stimulante pour le « il» du roman ${ }^{15}$. Pour ce jeune homme, les pommes sont par conséquent un objet à cacher, et le soulagement qu'il éprouve après la lecture de la légende "Comment des enfants ont joué ensemble au boucher", semble aboutir à une légitimation de ses pratiques jugées coupables, puisque la pomme qu'il aurait prise, lui aussi, est associée à ces mêmes pratiques. Le roman conclut par un long paragraphe dans lequel le jeune homme se demande qui il est, et s'il est bien lui-même ou quelqu'un d'autre. Le roman présente une lecture atypique de la légende, puisque même le sentiment d'innocence ne semble pas épargner une mort symbolique au personnage principal lorsqu'il se demande s'il est « ce jeune homme dont le corps à demi dévêtu avait été retrouvé aux environs de la petite ville " (Le Miroir quotidien, p. 143). Là encore, le lecteur est incité par l'auteur à combler un blanc du récit: pourquoi l'innocence n'est-elle pas de nature à calmer le 
personnage et à le conforter dans son identité ? Nous pensons qu'il s'agit, là encore, d'un renvoi lointain et certes symbolique à la situation de persécution raciale qui constitue l'arrière-plan non explicité du roman.

\section{Les métamorphoses du personnage : une référence aux contes?}

Ce procédé de « réécriture » nous paraît d'autant plus remarquable que la scène finale du Miroir quotidien se trouve dans des passages où l'auteur évoque l'époque qui sert de toile de fond au récit Un Jardin en Allemagne, avant son départ pour l'Italie et la France. Goldschmidt s'efforce de restituer une perception du monde qu'imprègnent certains éléments du conte. Dans la perception de l'enfant, c'est le caractère d'inquiétante étrangeté et un monde quasi-animiste entourant la maison natale qui prévalent sur le merveilleux du conte. Mona Körte, dans un article suggestif intitulé « Devenir animal » [ Tier-Werden], a expliqué le motif de la métamorphose, mobilisé ici par Goldschmidt, par la référence à la lecture de $\mathrm{Kafka}^{16}$. Sans contester une inspiration puisée dans l'univers de Kafka, il nous semble plus pertinent de fonder l'analyse sur des rêves enfantins dont Goldschmidt fait le récit dans son autobiographie. L'ambiguïté réside dans le fait que la métamorphose est à la fois menaçante et potentiellement libératrice puisque c'est l'«identité » du personnage telle qu'elle est désignée par les lois de Nuremberg, qui le rend « coupable » en raison de ses origines. Quitter son corps, être quelqu'un d'autre et non pas celui qu'il est, serait capable de le sauver. Dans son autobiographie La Traversée des fleuves, Goldschmidt relate ses angoisses au réveil matinal, sa peur d'être « jeté » dans un corps de cheval :

Souvent, je me réveillais le matin contracté par la terreur qu'il s'en était fallu de peu que je ne sois cheval, comme si je pouvais encore basculer hors de moi, dans une vie ronde, muette, à ras de terre. J'aurais pu aussi bien être jeté dans un corps sans bras. C'était à en suffoquer d'angoisse. Je racontais alors à ma mère ce genre de rêveries et elle les complétait, comme si elle les avait en partie vécues dans son propre corps. Mère et fils participaient du même corps. (La Traversée des fleuves, p. 84)

La métamorphose est décrite sous un angle menaçant. Le cheval représente une existence réduite : le garçon y perdrait ses bras ainsi que la langue humaine et vivrait «à ras de terre ». L'on peut supposer que la transformation est une manière d'évoquer l'existence réduite que craignent les personnes dénommées «juives", sans en parler explicitement. L'enfant semble être au courant d'une menace qu'il n'arrive pas à cerner. Le texte ne précise pas comment la mère arrive à compléter ce que le jeune garçon lui raconte: partage-t-elle ce genre de craintes ou a-t-elle une compréhension intuitive de son fils ? Une des particularités des récits de Goldschmidt est justement de renoncer à expliciter les relations de cause à effet. Ces souvenirs apparemment dépourvus de portée historique marquent le début du chapitre "Une enfance coupable». De par leur position, l'auteur suggère qu'il y a un rapport entre ces cauchemars et la notion de culpabilité. Il nous parait légitime de supposer que ce souvenir d'enfance est à l'origine du motif de la métamorphose chez Goldschmidt.

Un jardin en Allemagne propose une variante de cette métamorphose angoissante (Un jardin en Allemagne, p. 32-35), beaucoup plus élaborée que les rêves évoqués dans La Traversée des fleuves. La scène commence par un semblant d'idylle, mais bascule rapidement et la 
métamorphose est avant tout perçue comme une menace existentielle. Alors que l'autobiographie se contente d'évoquer " des rêves angoissants », la métamorphose est ici déclenchée par un «on» anonyme qui transforme le protagoniste en cheval. L'animal garde des traits humains et se distingue par ses faiblesses et défauts physiques, ce qui nous invite à voir en lui une forme réduite comme emprisonnée de l'existence humaine.

Le temps est beau et le soleil déjà bas. Le cheval passé on se retourne sur lui : il est nu, d'une nudité humaine, constamment exposée à tous les regards, enfermé, suffocant, cheval sans bras.

Journellement presque, cette peur de retomber hors de soi: on n'est que provisoirement celui qu'on est, on va vous tirer de là pour vous mettre dans ce cheval, comme si on était emporté d'au-dessus, suspendu au bout de doigts qu'on ne voyait pas et qui vous mettraient où ils voudraient. (Un jardin en Allemagne, p. 32-33).

Nous souhaitons donc proposer une lecture de cette métamorphose fondée sur l'imaginaire du conte, où les personnages changent parfois d'apparence (que ce soit contre leur gré ou non), et où les animaux peuvent communiquer avec les hommes ou les comprendre. L'imaginaire enfantin semble imprégné par cette logique du conte au point de l'appliquer à son entourage, puisqu'il se croit percé à jour par les animaux: "Les animaux savent: ils ont vu l'enfant dans les buissons se mettre nu, s'étonnant d'être et d'avoir ce corps très lisse [...]» (Un jardin en Allemagne, p. 33-34). Dans cette scène, la présence des animaux n'est pas de nature à calmer l'enfant craignant les regards des adultes; au contraire, ce sont les animaux qui prennent potentiellement le rôle de ses persécuteurs. L'enfant s'enfuit, puisqu'il est persuadé que le chat l'attaquera. Il y croit avec tant de conviction qu'il sent déjà la présence physique du chat en train de l'attaquer :

Quand cette image [d'un martyre représenté nu, en l'occurrence le Christ] arrivait, le moindre craquement dans les buissons le faisait transpirer d'angoisse, lui gelait le corps, ce n'était jamais personne, un animal, un chat au regard vert, soudain là comme depuis toujours et qui avait tout $v u$.

Le chat va le griffer, se plaquer contre lui, se mouler à lui, sans qu'il puisse le détacher, il va lui enfoncer ses griffes dans le visage : il en sent la touffeur obscure et sèche lui couvrir les yeux et, criant d'horreur, il court à travers les ronces. (Un jardin en Allemagne, p. 34)

La scène, que nous n'allons pas reprendre en entier, mêle la fascination pour la nudité (représentée par l'enfant ainsi que par un cheval pommelé) à l'image du martyre. Elle préfigure aussi l'abus que l'adolescent subira lorsqu'il sera accueilli dans un pensionnat de Haute-Savoie ${ }^{17}$. La métamorphose se termine par un moment apparemment paisible, mais dans lequel le «wagon» et la " plaine immobile » peuvent être interprétés comme une allusion à la déportation :

[...] il laissait son regard courir en avant sur le sol plat, fermé sous la tranchée sombre prise dans la clarté alentour, couverte de feuilles jaunes et ocre des automnes précédents, l'été pénétrait çà et là par des trouées rondes, tremblantes que les bouffées de vent ouvraient et fermaient. Des bruits arrivaient de la plaine et, entouré de cette voûte comme dans un wagon, il lui semblait rouler à travers la plaine immobile (Un jardin en Allemagne, p. 35).

Pour conclure, nous aimerions évoquer une allusion au "Petit Poucet» dans La Forêt interrompue qui s'écarte de la perspective adoptée ailleurs par Goldschmidt par rapport aux contes. Tandis que les soldats allemands montent à la ferme dans lequel le garçon est caché pour y acheter du beurre et des œufs, l'enfant reste immobile dans le foin et s'imagine transformé en poutre dans l'espoir de passer inaperçu (La Forêt interrompue, 
p. 29). L'autre idée qui semble le calmer, est de prendre la place du Petit Poucet, et de se laisser emporter par un énorme cheval protecteur :

[...] il aurait suffi de se réfugier dans l'oreille du cheval, comme dans le conte de Grimm, il s'y serait accoudé et, protégé par la voûte du pavillon de l'oreille audessus de lui, il aurait vu défiler les paysages, à l'abri de la pluie, des orages, il aurait entendu, autour de ce corps qui n'en finissait pas, les voix qui le cherchaient pour l'emmener loin dans les plaines de l'Est. (La Forêt interrompue, p. 30)

À notre connaissance, c'est l'unique occurrence chez Goldschmidt d'une scène issue d'un conte qui symbolise un sentiment de sécurité : le jeune pensionnaire caché s'imagine être protégé par un cheval ${ }^{18}$. Réduit aux dimensions du Petit Poucet, il pourra à la fois disparaître et continuer à exister dans un corps humain. Face à ses agresseurs, incarnés par les soldats allemands, le cheval est un personnage adjuvant. Caché dans son oreille, le garçon trouvera un refuge sûr, lui permettant de rester en contact avec le monde qui l'entoure, notamment à travers les paysages qu'il voit.

Dans son essai Une forêt et ses lisières, Goldschmidt explore le terme d' "unheimlich " (l'inquiétante étrangeté), qu'il place au cœur de l'enfance et qu'il relie expressément aux contes des frères Grimm :

Das Unheimliche se situe donc bel et bien au cœur du monde de l'enfance et il est curieux [...] que Freud passe littéralement d'un trait de plume (Das Unheimliche, p. 160) sur les contes de Grimm, allant jusqu'à trouver chez Hauffman, un conteur parfois mièvre, ce qu'il ne voit pas chez Grimm : à savoir ces membres coupés et ses têtes tranchées qui sont, de même que les poupées soudain animées, l'un des éléments constitutifs de l'Unheimlich : or ces contes existent chez Grimm et ils sont toujours liés à un secret. (Une forêt et ses lisières, p. 13)

Par cette définition personnelle de l'«Unheimlich», de l'inquiétante étrangeté caractérisant à la fois l'enfance et les Contes des frères Grimm, nous souhaitons rappeler que les textes de Goldschmidt mêlent souvent les genres et que l'autobiographie au sens large du terme peut réapparaître, d'une manière ou d'une autre, dans un essai, comme dans cette définition, en cohérence avec son propre vécu tel qu'il le présente dans ses fictions et dans son autobiographie La Traversée des fleuves.

\section{BIBLIOGRAPHIE}

DEMISCH Heinz (2003), Ludwig Richter 1803-1884. Eine Revision [Ludwig Richter 1803-1884. Une révision], Berlin: Gebrüder Mann Verlag.

EISSLER Kurt R. (1963), « Die Ermordung von wie vielen seiner Kinder muß ein Mensch symptomfrei ertragen können, um eine normale Konstitution zu haben? » [Le meurtre de combien de ses enfants un parent doit-il supporter sans présenter des symptômes pour avoir une constitution normale ?], Psyche, 17 (cahier 5), 241-291.

GOLDSCHMIDT Georges-Arthur (1981), Le Miroir quotidien. Roman, Paris : Le Seuil.

GOLDSCHMIDT Georges-Arthur (1982), « Une forêt et ses lisières ", L'Écrit du temps. Langues

familières, langues étrangères (11-33), $\mathrm{n}^{\circ} 2$. 
GOLDSCHMIDT Georges-Arthur (1986), Un jardin en Allemagne. Récit, Paris : Le Seuil.

GOLDSCHMIDT Georges-Arthur (1991), La Forêt interrompue. Récit, Paris : Le Seuil.

GOLDSCHMIDT Georges-Arthur (1991), Die Absonderung. Erzählung. Mit einem Vorwort von Peter Handke, Zürich : Ammann [La Ligne de fuite. Traduit par Jean-Luc Tiesset, Paris, Flammarion, 1994].

GOLDSCHMIDT Georges-Arthur (1999), La Traversée des fleuves. Autobiographie, Paris : Le Seuil.

GOLDSCHMIDT Georges-Arthur (2008), Des Pudels Kern. Ein Gespräch. Antworten auf Fragen von Tim Trzaskalik [Au cœur du sujet. Un entretien. Réponses aux questions de Tim Trzaskalik], Berlin : Matthes \& Seitz.

GRIMM Jacob et Wilhelm, Contes pour les enfants et la maison, collectés par Les Frères Grimm, édités et traduits par Natacha Rimasson-Fertin (2009), Paris : éditions José Corti, 2 vol.

KÖRTE Mona (2007), « Tier-Werden. Verwandlungsangst in Georges-Arthur Goldschmidts

Autobiographie Über die Flüsse » [Devenir animal. La peur de la métamorphose dans

l'autobiographie de Georges-Arthur Goldschmidt La Traversée des Fleuves], T. Trzaskalik (dir.),

Kultur \& Gespenster [Culture \& Spectres], 5, Georges-Arthur Goldschmidt, 120-129.

UTHER Hans-Jörg (2008), Handbuch zu den „Kinder- und Hausmärchen“ der Brüder Grimm : Entstehung Wirkung - Interpretation [Manuel des Contes pour les enfants et la maison des frères Grimm : genèse - retentissement - interprétation], Berlin : De Gruyter.

\section{NOTES}

1. «Il ne faut pas oublier, en effet, que le nazisme fut aussi et peut-être avant tout une entreprise d'éradication de l'Allemagne elle-même. » (La Traversée des fleuves, p. 100)

2. Dans une longue interview, parue sous le titre Des Pudels Kern [Au cœur du sujet], l'auteur parle de «die deutsche Obsession des Judentötens bei Luther, Kant, Fichte, Schopenhauer und dem NaziHeidegger, das ist bei denen ein richtiger Trieb » [l'obsession allemande de tuer les Juifs chez Luther, Kant, Fichte et le nazi Heidegger, c'était une vraie pulsion chez eux (ma traduction)] arguant un peu plus loin, dans une référence à une sorte d'inconscient collectif de la nation allemande, imprégné par le luthéranisme: «die Nationalsozialisten [realisierten] einen unbewußten deutschen Wunsch [...], mit dem endlich die den Deutschen von Luther eingepaukte Schuld aufgehoben wäre » [les national-socialistes ont réalisé un désir de l'inconscient allemand, potentiellement capable d'effacer la culpabilité inculquée aux Allemands par Luther (ma traduction)] (Des Pudels Kern, p. 146 et 147). Dans son autobiographie, Goldschmidt écrit que le nazisme était « une dimension intime, presque corporelle » de l'Allemagne, et qu'il retrouvait cette dimension dans les textes allemands qu'il découvre après la libération grâce à « des polycopiés en vue de l'agrégation » : «Ces textes détonnaient, pleins de cette langue raide et froide où tout se disait efficacement. Pour moi, l'Allemagne gymnaste et forestière devenait à la fois gênante et étrangère ; rien ne m'en étonnait. [...] Ces textes étaient tous compacts, sans alinéas, serrés et de pensée dense qui, traduite, était souvent creuse. » Il fait aussi référence au respect voué aux autorités ainsi qu'à sa dimension politique, et conclut de manière intransigeante : «D'un coup je les connus tous, ces Gundolf, ces Korff, ces Spengler et autres naïfs prolixes. Je sus d'emblée à quoi j'avais affaire, à d'énormes paquets de mots, épais, confus et trop graves pour être pris au sérieux. " (La Traversée des fleuves, p. 210-211)

3. Ludwig Richter (1803-1884), peintre romantique, connu du grand public pour ses illustrations de contes, a illustré, entre autres, Die Volksmärchen der Deutschen par Karl August Musäus, édité 
par J. L. Klee à Leipzig en 1842, et le Deutsches Märchenbuch de Ludwig Bechstein paru à Leipzig en 1853, son sujet de prédilection selon Heinz Demisch. (H. Demisch : Ludwig Richter 1803-1884. Eine Revision, 2003, p. 215 et références bibliographiques p. 416 et 422.) Une myriade d'illustrations de contes, mettant en scène « Le Petit Chaperon rouge ", "Blanche-Neige » et " Le Petit Poucet », et pour la plupart destinées au recueil de Bechstein, témoigne de sa productivité et de la popularité dont il jouit auprès de son jeune public. Toujours selon Demisch, il n'existe pas de recueil des Contes de Grimm illustré par Richter: «Es gibt von Richter illustrierte Märchensammlungen nach Musäus, Bechstein, Andersen, Colshon und Renick, es gibt aber keine von Richter illustrierte Sammlung Grimmscher Märchen. "[Richter a illustré des recueils de contes d'après Musäus, Bechstein, Andersen, Colshon et Renick, mais on ne trouve pas de recueil de contes des Frères Grimm illustrés par Richter (ma traduction)] (H. Demisch, art. cité, p. 37).

Cette précision de Demisch ne désavoue pourtant pas la mémoire de Georges-Arthur Goldschmidt. Si Richter n'a jamais illustré le recueil des Kinder- und Hausmärchen en tant que tel, on trouve tout de même des éditions de contes choisis parues avec ses illustrations. Il nous paraît difficile de donner une édition de référence puisque ses éditeurs qui détenaient les droits d'auteur avaient l'habitude de reprendre ses illustrations dans d'autres éditions (Demisch, ouvr. cité, p. 36-38). Nous aurions voulu vérifier dans la thèse de Regina Freyberger: Märchenbilder-Bildermärchen: Illustrationen zu Grimms Märchen 1819-1945. Über einen vergessenen Bereich der deutschen Kunst, 2009, mais le livre est actuellement en cours de catalogage à la Bibliothèque nationale. Le choix éditorial de L'école des loisirs de présenter Les Contes pour les enfants et les parents accompagnés des illustrations de Ludwig Richter montre peut-être à quel point, pour la postérité, les illustrations de Ludwig Richter sont restées associées aux Contes des frères Grimm (voir Grimm, Jacob et Wilhelm : Contes pour les enfants et les parents. Textes intégraux traduits par Max Buchon illustrés par Ludwig Richter, 3 tomes, Paris, L'école des loisirs, 1979-1980).

4. «Derjenige, um den es hier geht, ist der Sohn einer der ältesten jüdischen, dann zum Protestantismus konvertierten Familien Hamburgs. Bis ins innerste Wesen mit der Deutschheit verbunden, lebte diese Familie wie eine deutsche Familie aus dem Bilderbuch, sozusagen mit Ludwig Richter, Hans Thoma und den Gebrüdern Grimm. » (Ein Leben zwischen Frankreich und Deutschland, p. 27) [Celui dont il est question ici, est le fils d'une des plus vielles familles juives d'Hambourg, convertie ensuite au protestantisme. Liée à la germanité jusque dans leur être le plus intime, cette famille vécut comme une famille allemande modèle, pour ainsi dire en compagnie de Ludwig Richter, de Hans Thoma et des frères Grimm. (Ma traduction.)]

5. Par ailleurs, les scènes de lecture réellement racontées sont tout sauf idylliques; dans La Forêt interrompue, la mère du personnage principal s'enferme avec son fils et lui lit des «contes de Grimm » pour éviter qu'il ne découvre son père en train de peindre «le $\mathrm{Nu}$ » [sic !], tandis que l'enfant, ayant bien compris que cette lecture n'est qu'un prétexte pour le tenir à l'écart de la nudité, est obsédé par les images du corps nu qui lui est caché. Il s'agit d'un modèle peint par son père qui déclare vouloir s'exercer à ce genre de peinture tandis que sa femme s'oppose à cette situation. (Un jardin en Allemagne, p. 18-19)

6. Voir par exemple à la page 42 de La Traversée des fleuves : «Le nazisme était d'autant plus tragique pour tous ces «non-Aryens » allemands qu'ils avaient été allemands avec une ferveur presque religieuse, au point d'être souvent atteints de ce fameux jüdischer Selbtsthass (haine de soi juive). »

7. Voici un des exemples les plus parlants de cette substitution puisque le tabou de la sexualité est évoqué par l'expression vague "das andere ", non explicitée par la suite. À ce moment du récit, le jeune garçon se trouve dans le train qui l'emmènera à Munich avant de quitter l'Allemagne pour Florence: «Im Zug, seltsamem viereckigem Kasten, in dem er saß, während die Landschaft draußen an ihm vorbeizog, hatte er auf einmal gewußt: wäre er wirklich ein Jude gewesen, er hätte es nie sagen dürfen, wie er auch das andere nie sagen durfte. » (Die Absonderung, p. 16-17) [Dans le 
train, étrange boîte quadrangulaire où il demeurait assis tandis que paysage défilait dehors, il avait eu tout à coup la certitude que, s'il avait été juif pour de bon, il n'aurait jamais dû le dire, pas plus qu'il ne devait jamais dire le reste.] (La Ligne de fuite, p. 16).

8. Kurt R. Eissler, dans un article militant intitulé « Die Ermordung von wievielen seiner Kinder mu $\beta$ ein Mensch symptomfrei ertragen können, um eine normale Konstitution zu haben?» [Le meurtre de combien de ses enfants un parent doit-il supporter sans présenter de symptômes pour avoir une constitution normale? (Ma traduction)], paru dans Psyche, 17, 1963, p. 241-291, est un des premiers à évoquer la possibilité que le masochisme ait un rôle protecteur dans une situation de persécution extrême. Il s'agit d'une pure hypothèse émise lorsqu'il cherche un facteur potentiellement capable de sauver certains survivants des camps d'extermination d'un traumatisme qui les marquera à vie, alors que tout son article tente de prouver qu'a priori personne ne peut en sortir indemne. Eissler n'exclut pas que la psychanalyse parviendra peutêtre un jour à prouver que «un grand nombre de personnes est protégé de dommages psychologiques durables par la mobilisation de sources masochistes très profondes » ( [ [ass] eine große Anzahl von Menschen durch Mobilisierung tiefster masochistischer Quellen vor Dauerschädigungen des seelischen Apparates geschützt wird»), art. cit., p. 288.

9. «[...] er hatte noch von zu Hause einige Bücher mit schönen Bildern mitnehmen können: „Märchen“, „Erzählungen“, „Im Wald und auf der Heide“ von Hermann Löns, das Leben des Johann-Hinrich Wiechern [ sic !] [...]. In seinem Fach durfte er auch Hefte, Federhalter und ein Watermantintenfaß unterbringen und ein immer mehr abgeschabtes Märklinspielzeugauto aufbewahren. Er strich manchmal mit der Hand darüber, vielleicht genügte es, die Augen dabei zu schließen, um noch zu Hause zu sein. » (Absonderung, p. 66-67)

10. Il nous paraît improbable de supposer que les parents n'aient choisi que des livres d'un seul auteur pour leur fils, et nous excluons pour cette raison qu'il s'agit des contes de Löns, d'autant plus qu'ils ne sont cités nulle part dans les récits de Goldschmidt et que Löns, chantre du terroir, a été instrumentalisé par les nazis.

11. La cruauté des évènements racontés serait la raison essentielle de la suppression de la légende "Comment des enfants ont joué ensemble au boucher » des éditions ultérieures. Uther suppose que les frères Grimm, qui avaient pourtant défendu leur choix, ont finalement retiré cette légende parce qu'ils craignaient que l'action du conte ne serve de mauvais exemple. En revanche, «La main au couteau » a probablement été supprimé du recueil du fait de ses origines étrangères, précisément écossaises, et en raison de sa fin malheureuse et de la crainte que l'usage du mot Liebhaber [amant] soit ressenti comme trop osé pour de jeunes enfants. (H.-J. Uther, 2008 : 423 et 425$)$.

12. On conçoit aisément que la traduction donnée est de Goldschmidt. Par ailleurs, les trois contes en question ne figurent pas dans le choix de contes traduits par Marthe Robert (Grimm : Contes. Choix, traduction et préface de Marthe Robert, Paris, Gallimard, 1967). La traduction d'Armel Guerne se base sur la toute dernière édition des Contes parue en 1857 (Ausgabe letzter Hand), et ne donne de version française que de «L'Enfant difficile " [autre traduction de «L'Enfant entêté » (KHM 117)] (Jacob et Wilhelm Grimm : Les contes. Kinder- und Hausmärchen. Texte français et présentation par Armel Guerne, Paris, Flammarion, 1967).

13. «Cela avait déjà été : les soirs ainsi se rejoignaient. On se levait et on pouvait montrer ses mains, et c'était le matin d'été, et on ne lui ferait pas de reproches. » (Le Miroir quotidien, p. 57)

14. Ce sujet est prédominant dans le récit Die Absonderung et Die Aussetzung [L'Abandon (ma traduction)] et Le Miroir quotidien l'évoque souvent par rapport à d'autres personnages (littéraires ou autres), dont le « il » du texte se sent proche, par exemple à travers un tableau de Reaburn au Louvre, mettant en scène Madame Blichwood et son jeune fils Charles, élève à Eton, sur lequel on peut apercevoir au fond un "bosquet de bouleaux aux troncs blancs et dont les longues branches souples descendaient à grandes courbes vers le sol, jusqu'à portée de main ». À la différence de ses autres récits, Goldschmidt fait dans Le Miroir quotidien très explicitement référence à un certain 
courant littéraire : «Chaque fois la même secousse le parcourait. Il savait ce qui se passait à Eton vers 1800, il avait lu la biographie de Shelley [...], il avait lu Swinburne. Il était CELUI QUI SAVAIT CE QUI L'ATTENDAIT. Il était Herbert Seyton, il était Walter Lunsford, il était Reginald Harewood. Il habitait Kirklowes: Était-ce à lui que le roi George III passant à cheval, à proximité, avait demandé quand avait lieu le dernier mariage entre sa personne et les bouleaux du parc Eton?» ( Le Miroir quotidien, p. 139)

15. « Il [...] avait posé [sur la chaise], fraichement cueillies, les branches écotées auxquelles pourtant il laissait les bourgeons de l'extrémité. Recouvertes d'une serviette, on ne les verrait pas et il avait pris l'aisance des gestes longuement répétés. Souvent il s'était assis pour creuser ses pommes achetées au marché parce qu'on ne l'y connaissait pas. Il choisissait des pommes rouges du Canada car elles ne cassaient pas. » (Le Miroir quotidien, p. 140)

16. Mona Körte : « Tier-Werden », p. 122-123.

17. «On est cheval-tête pris entre les cuisses nues du cavalier qui enserrent le visage: une douceur chaude contre les joues, résistante et souple. » (Un jardin en Allemagne, p. 33).

18. Il s'agit du conte « Daumesdick » (KHM 37), qui porte le titre " Gros-comme-le pouce » dans la traduction des Contes des frères Grimm par N. Rimasson-Fertin (Grimm, 2009, vol. 1 : 221-228).

\section{RÉSUMÉS}

«Je retrouvais les paysages de contes de Grimm à ma porte, la forêt sombre, les étangs mystérieux, entourés d'arbres aux épais feuillages, et en hiver le dessin étrange des branches, sans cesse agitées par le vent. Un effroi indéfinissable habitait désormais cette campagne si calme, doucement étendue. Partout on pouvait y être mis à mort. » (La Traversée des fleuves, p. 111)

La menace de la mort est un des éléments constitutifs de l'enfance de Georges-Arthur Goldschmidt, que l'auteur ne cesse de raconter dans ses récits jusqu'alors peu étudiés par la critique. Tout comme la Bible et ses illustrations, les contes des frères Grimm, illustrés par le même Ludwig Richter, constituent une des lectures fondatrices de l'imaginaire enfantin. Tandis que la Bible, dans lequel le jeune garçon découvre à la fois Jésus et le mot «juif» ainsi que la figure du martyre qui le préoccupe des années durant, prétend à l'universalité, les contes des frères Grimm plongent le jeune «Jürgen Arthur » au cœur d'une culture germanique dont il sera exclu par les lois de Nuremberg.

Tout d'abord, la lecture des contes établit un lien avec le passé littéraire du XIX ${ }^{\mathrm{e}}$ siècle et permet de mettre en scène l'arbitraire de la menace de mort dont il est la cible. Ensuite, le livre est un des rares objets qu'il emporte avec lui au moment de la séparation avec ses parents qui se révèle être définitive. Le Miroir quotidien, le premier récit de Goldschmidt, accorde une place centrale à l'un des contes des frères Grimm : grâce à la lecture du conte «Comment des enfants ont joué ensemble au boucher ", traduit par Goldschmidt et inséré intégralement dans son récit, le protagoniste est libéré de son sentiment de culpabilité. Au conte succède une longue vague de questionnements relative à «la honte»: la lecture du conte déclenche une forte réaction psychique chez le personnage qui fait l'objet d'un récit propre sans qu'on puisse parler d'« adaptation » du conte au sens propre.

Un procédé de «réécriture " qui me paraît d'autant plus remarquable que la scène finale du Miroir quotidien se trouve dans des passages où l'auteur évoque l'époque qui sert de toile de fond 
au récit Un Jardin en Allemagne, avant son départ pour l'Italie et la France. Goldschmidt s'efforce de restituer une perception du monde imprégnée par l'imaginaire du conte. Mona Körte, dans un article suggestif intitulé « Tier-Werden », a expliqué le motif de métamorphose, mobilisé ici par Goldschmidt, par la référence à la lecture de Kafka. Sans contester une inspiration puisée dans l'univers de Kafka, il me semble plus pertinent de fonder l'analyse sur les fantasmes enfantins dont Goldschmidt fait le récit dans son autobiographie.

L'ambiguïté réside dans le fait que la métamorphose est à la fois menaçante et potentiellement libératrice puisque c'est l'«identité » du personnage telle qu'elle est désignée par les lois de Nuremberg, qui le rend « coupable » en raison de ses origines. Quitter son corps, être quelqu'un d'autre et non pas celui qu'il est, serait capable de le sauver. Dans la perception de l'enfant, c'est le caractère «Unheimlich » et un monde quasi-animiste entourant la maison natale qui prévalent sur le merveilleux du conte. Une lecture d'un essai de Goldschmidt explorant le terme « Unheimlich », qu'il place au cœur de l'enfance et qu'il relie expressément aux contes des frères Grimm clôturera mes réflexions.

"I could find the scenery of Grimm's tales on my doorstep, the dark forest, the mysterious ponds lined by the thick foliage of trees and in the wintertime the strange pattern of branches relentlessly battered by the wind. An unscrutable tremor inhabited this countryside that was so calmly rolling away. And yet, everywhere, you could be put to death." (La Traversée des fleuves, p. 111) [my translation]

The threat of death is constitutive of Georges-Arthur Goldschmidt's childhood, which the author continually depicts in his narratives. Until now, these works have rarely been subjected to critical study. Just like the Bible (and the Bible's illustrations), the Children's and Household Tales of the Grimm Brothers, both illustrated by Ludwig Richter, are to be seen as fundamental texts of the child's psyche. While the Bible, in which the small boy discovers Jesus and the word "Jew" at the same time, as well as the figure of the martyr which subsequently haunts him, aspires to universality, the Grimms' fairy tales throw the young "Jürgen-Arthur" right into the heart of German culture, from which he will later be excluded by the Nuremberg Laws.

At first, reading the fairy tales establishes a link with the literary past of the 19th century and reveals that the protagonist is under an arbitrary threat. Then, the book is one of the rare objects which he takes with him when he is being separated from his parents, a separation which proves to be definitive. Le Miroir quotidien (The Everyday Mirror), Goldschmidt's first novel, places one of the Grimms' tales "Wie die Kinder Schlachtens miteinander gespielt haben" in a central position: The reading of the tale, translated by Goldschmidt and integrally inserted into the novel, frees the protagonist from his feeling of guilt. A long stream of questions connected with "shame" follows this tale: the reading releases a strong psychic reaction from the character who is the object of a distinctive narrative rather than an actual adaptation. A process of "rewriting" which seems to me all the more remarkable as the final scene of the Miroir quotidien calls to mind the period which serves as a backdrop to the narrative Un jardin en Allemagne (A Garden in Germany), before his departure to Italy and France. Goldschmidt strives to recreate a perception of the world impregnated by the universe of fairy tales. Mona Körte, in a thought-provoking article entitled "Tier-Werden", has explained the motif of metamorphosis as being a reference to Kafka. Without contesting a certain amount of inspiration derived from the universe of Kafka, I will suggest that it might be more relevant to base the interpretation of this text on the childhood fantasies narrated by Goldschmidt in his autobiography.

The metamorphosis is both threatening and potentially liberating, because the way how the "identity" of the person is being described by the Nuremberg Laws, makes the person "guilty" because of his or her origins. Leaving his body, being someone else and not the one he is, might possibly save him. In the children's perception, the character of "unheimlich" and a quasianimistic world around the house of his family prevail over the magical of the fairy tale. A 
lecture of an essay by Goldschmidt exploring the term "unheimlich", which he places right at the center of the childhood and which relates explicitly to the Grimm's fairy tales will close my reflections.

Die Landschaften der Grimmschen Märchen lagen vor meiner Tür, der dunkle Wald, die ein wenig unheimlichen Teiche, von alten Bäumen umgeben, und im Winter die seltsamen Zeichnungen, die die vom Wind bewegten Äste in den Himmel warfen. Eine Unruhe, ein Schrecken lag aber von nun an auf dieser ruhigen, ausgebreiteten Landschaft, man konnte da überall totgeschlagen werden. (Über die Flüsse, S. 128)

Die Bedrohung seines Lebens ist eines der konstitutiven Elemente der Kindheit von GeorgesArthur Goldschmidt, um die der Autor in seinen Erzählungen, die bisher verhältnismäßig selten zum Gegenstand der Forschung geworden sind, immer wieder kreist. Die Märchen der Gebrüder Grimm stellen genau wie die Bibel in einer von Ludwig Richter illustrierten Fassung eine Lektüre dar, die die kindliche Vorstellungswelt prägt. Während die Bibel, in der der kleine Junge zugleich Jesus und das Wort "Jude“ entdeckt, sowie die Figur des Märtyrers, die ihn lange Jahre beschäftigen wird, einen universellen Anspruch vertritt, taucht der junge "Jürgen Arthur" mit den Märchen der Gebrüder Grimm ganz in die deutsche Kultur ein, aus der er durch die Nürnberger Gesetze ausgeschlossen wird.

Das Lesen der Märchen stellt zunächst einen Zusammenhang mit der literarischen Vergangenheit des 19. Jahrhunderts her und erlaubt nebenbei auch, die Willkürlichkeit der Bedrohung seines Lebens darzustellen. Das Buch ist auch einer der seltenen Gegenstände, die der Protagonist im Moment der Trennung von seinen Eltern, die sich als endgültig erweisen wird, mitnehmen kann. In der ersten neueren autofiktionalen Erzählung von Goldschmidt, Le Miroir quotidien, nimmt eines der Grimmschen Märchen eine zentrale Stellung ein: der Protagonist wird beim Lesen des Märchens „Wie die Kinder Schlachtens miteinander gespielt haben“, das Goldschmidt übersetzt und vollständig in den Text eingefügt hat, von seinem Gefühl der Schuld befreit. Auf das Märchen folgt eine lange Reihe von Fragen, die sich auf die Scham beziehen: Das Lesen des Märchens löst eine starke psychische Reaktion der Hauptfigur aus, die Gegenstand eines eigenen Berichts wird, ohne dass man von einer Adaptation oder Bearbeitung im engeren Sinne sprechen kann.

Ein Verfahren des Umschreibens, das mir bemerkenswerter erscheint, als diese Schlussszene des Miroir quotidien, findet sich in den Passagen von Ein Garten in Deutschland, in denen der Autor die Zeit vor seinem Aufbruch nach Italien und Frankreich schildert. Goldschmidt unternimmt hier den Versuch, eine Wahrnehmung der Welt zu rekonstruieren, die von der Welt der Märchen geprägt ist. Mona Körte hat in dem anregenden Artikel „Tier-Werden“ das Motiv der Metamorphose, das Goldschmidt hier einsetzt, als Referenz auf seine Kafka-Lektüre zurückgeführt. Ohne eine Inspiration durch die Welt Kafkas zu bestreiten, erscheint es mir naheliegender, für die Analyse von den kindlichen Phantasien auszugehen, über die Goldschmidt in seiner Autobiographie berichtet. 


\section{INDEX}

Schlüsselwörter : Gebrüder Grimm, Kinder- und Hausmärchen, Adaptation, Umschreiben, Georges-Arthur Goldschmidt, Verfolgung, Nürnberger Gesetze, Zeugenschaft, Schuldgefühl, Metamorphose, Kafka

Keywords : Grimm Brothers, Child and Household Tales, adaptation, rewriting, Georges-Arthur Goldschmidt, persecution, Nuremberg Laws, testimony, sense of guilt, metamorphosis, Kafka Mots-clés : frères Grimm, Les contes pour les enfants et pour la maison, réécriture, adaptation, Georges-Arthur Goldschmidt, persécution, Lois de Nuremberg, témoignage, culpabilité, métamorphose, Kafka

\section{AUTEUR}

\section{KATJA MEISTER}

Freie Universität Berlin - université Paris Diderot / Paris 7 